\title{
INCIDÊNCIA DE FUNGOS TERMORRESISTENTES E PROPRIEDADES MICROBIOLÓGICAS DA POLPA DE CUPUAÇU CONGELADA (Theobroma grandiflorum Schum)
}

\author{
Marcela Vicente Vieira Andrade Gonçalves \\ Mestre em Produção Vegetal UENF \\ marcelandrade14@yahoo.com.br
}

Janine Passos Lima da Silva

Doutor em Ciências de Alimento/ UNICAMP/ Embrapa Agroindústria de Alimentos janine.passos@embrapa.br

\author{
Amauri Rosenthal \\ Doutor em Ciências de alimento/UNICAMP / Embrapa Agroindústria de Alimentos \\ amauri.rosenthal@embrapa.br
}

\section{Angela Aparecida Lemos Furtado}

Doutor em Tecnologia de Processos Químicos e Bioquímicos/ UFRJ / Embrapa

Agroindústria de Alimentos

angela.furtado@embrapa.br

\section{Veronica Maria de Araújo Calado}

Doutor em Engenharia Química/UFRJ / Universidade Federal do Rio de Janeiro - Escola de Química

calado@eq.ufrj.br

\section{RESUMO}

Cupuaçu, também conhecido como cupu, cupuassu ou cupu-do-mato é uma das principais e mais consumidas frutas da região amazônica brasileira. O cupuaçu é uma fruta ácida, de sabor exótico e agradável, rico em compostos voláteis e sais minerais, pertence à família Sterculiacea, cuja denominação binomial é Theobroma grandiflorum schum. Esta fruta apresenta crescente utilização no Brasil para o mercado interno e externo, sendo comumente comercializada como polpa congelada. A polpa, que tem aroma e sabor muito apreciados, é utilizada para sucos, refrescos, sorvetes, doces, compotas, licores, iogurtes, concentrados, polpas desidratadas e aromatizantes. Apesar de sua importância, há poucos dados de caracterização e contaminação da polpa de cupuaçu. Portanto, amostras de polpa de cupuaçu foram avaliadas quanto a contagem total de bolores e leveduras, Coliformes a 35 e $45{ }^{\circ} \mathrm{C}$, Salmonella sp e ocorrência de fungos termorresistentes. Os resultados demonstram a necessidade de aprimorar as boas práticas agrícolas e de fabricação e a implantação de sistemas de controle de qualidade para minimização dos riscos associados à contaminação por microrganismos.

Palavras-chave: Microrganismo, Termorresistência, Deterioração, Pasteurização. 


\begin{abstract}
Cupuaçu, also known as cupu, cupuassu or cupu of the woods is one of the leading and most widely consumed fruit in the Brazilian Amazon region. The cupuaçu is an acid fruit, of exotic and nice flavour, rich in volatile compounds and mineral salts, belonging to the family Sterculiacea whose binomial name is Theobroma grandiflorum Schum. This fruit has a growing use in Brazil for domestic and foreign markets, and is commonly marketed as frozen pulp. The pulp, which has aroma and flavor much appreciated, is used for juices, soft drinks, ice cream, candies, jams, liqueurs, yogurt, concentrated, dehydrated pulps and flavoring. Despite its importance, there are few data about characterization and contamination of cupuaçu pulp. Therefore, cupuaçu pulp samples were evaluated for the total count of yeasts and molds, Coliforms at 35 and $45^{\circ}$ C, Salmonella sp and occurrence of heat resistant fungi. The results demonstrate the need to enhance good agricultural and manufacturing practices and the implementation of quality control systems to minimize the risks associated with contamination by microorganisms.
\end{abstract}

Keywords: Microorganisms, Heat resistance, Deterioration, Pasteurization.

\title{
1. INTRODUÇÃO
}

Dentre as frutas tropicais nativas da Amazônia, o cupuaçu (Theobroma grandiflorum Schum) é aquela que reúne as melhores condições de aproveitamento industrial. É uma fruta originária do Sul e do Sudeste da Amazônia e é apreciada por sua polpa ácida e de aroma intenso. A polpa de fruta tem grande importância como matéria-prima, podendo ser produzida nas épocas de safra, armazenadas e processadas nos períodos mais propícios ou segundo a demanda do mercado consumidor (BUENO, 2002).

O cupuaçu vem conquistando o mercado de outras regiões do Brasil e despertando o interesse de países da Europa e da Ásia, sobretudo Inglaterra, Japão e Suécia, além dos Estados Unidos e países sul americanos. A parte do fruto mais aproveitada em termos comerciais ainda é a polpa, usada in natura na forma de suco ou como matéria-prima para fabricação de produtos derivados como cremes, tortas, sorvetes, néctares, balas, geléias, licores entre outros (YANG et al., 2003).

Os alimentos mais susceptíveis à deterioração por fungos termorresistentes são as frutas e seus derivados, como sucos, polpas, concentrados e frutas enlatadas. A principal fonte de contaminação das frutas é o solo, onde esses fungos estão presentes. A deterioração de produtos à base de frutas pode ser caracterizada pelo crescimento visível do fungo (FOSTER e VASAVADA, 2003). Estima-se que perdas pós-colheita variem de 15 a $50 \%$, dentro da media brasileira de perda pós-colheita de frutos tropicais no Brasil que se encontra na ordem de $30 \%$ dos produtos comercializados (XAVIER et al., 2009).

Devido a sua perecibilidade, não é adequado transportar o cupuaçu in natura por longas distâncias. Dessa forma, o congelamento da polpa é uma opção viável para evitar perdas de produção, pois assim preserva as características originais da fruta fresca possibilitando, inclusive, sua comercialização nos períodos de entressafra (FREIRE et al., 2009; MARTINS, 2008).

Logo, para possibilitar o transporte a longas distâncias, faz-se a pasteurização, que tem como principais objetivos a destruição de células vegetativas de microrganismos patogênicos, deteriorantes e a inativação enzimática da polpa (MARTINS, 2008). Embora a pasteurização estabilize o produto, sua qualidade final pode ser afetada, devido a perdas de compostos de aroma e sabor caracerísticos da fruta in natura. O efeito da temperatura contribui para a degradação da cor e para o escurecimento não enzimático (COSTA et al., 2003).

Constataram-se poucos dados na literatura sobre as carcaterísticas de qualidade da polpa de cupuaçu comercialmente disponível no país. Neste contexto, o objetivo desta pesquisa consistiu na caracterização microbiológica e também verificar a ocorrência de fungos termorresistentes, para então contribuir com informações para estabelecer um padrão de identidade e qualidade de polpa de cupuaçu. 


\section{MATERIAL E MÉTODOS}

Frutos in natura e polpa de cupuaçu, da safra 2008 e 2010 foram processados em uma indústria no Estado do Pará. As etapas do processamento são mostradas na Tabela 1.

Tabela 1. Amostras de frutos in natura e polpas congeladas de cupuaçu, safra 2008 e 2010 com suas respectivas codificações.

\begin{tabular}{cc}
\hline Etapas do processamento & Codificação \\
Frutos in natura & $\mathrm{F}$ \\
Polpa na recepção & $\mathrm{R}$ \\
Polpa após pasteurização & $\mathrm{P}$ \\
Polpa após envase & $\mathrm{E}$ \\
Polpa congelada armazenada (meia-vida de \\
seis meses)
\end{tabular}

Duas amostras foram retiradas ao final das etapas de despolpamento (polpa e semente após a quebra manual do fruto), homogeneização, pasteurização, envase, armazenamento (seis meses) e colocadas em sacos estéreis (Whirl-Pak ${ }^{\circledR}$ ). Em seguida, amostras não congeladas foram imediatamente levadas ao processo de congelamento em câmaras frigoríficas $\left(-40^{\circ} \mathrm{C}\right)$ na própria empresa, sendo somente removidas 24 horas após, e acondicionadas em caixas térmicas. Posteriormente, todas as amostras foram transportadas para a Embrapa Agroindústria de Alimentos, onde foram armazenadas congeladas em câmara frigorífica a $-18^{\circ} \mathrm{C}$ para posterior realização das análises.

\subsection{Análises microbiológicas}

As análises microbiológicas, em triplicata para Coliformes a $35^{\circ} \mathrm{C}$ e $45{ }^{\circ} \mathrm{C}$ e Salmonella sp, foram realizadas seguindo os padrões estabelecidos na RDC $n^{\circ} 12$ (BRASIL, 2001), com intuito de verificar as condições de higiene do processo, seguindo a metodologia do APHA (2010).

Para avaliar se a polpa estava em condições de ser consumida, foram realizadas análises em triplicata de contagem de leveduras, seguindo a metodologia do APHA (2010).

\subsection{Avaliação do binômio tempo-temperatura da pasteurização de polpa de cupuaçu}

A amostra de polpa de cupuaçu da safra de 2010, da etapa do processamento de homogeneização $(\mathrm{H})$ foi pasteurizada em trocador de superfície raspada (Armfield FT25D SSHE com sistema acoplado, utilizado na planta piloto da Embrapa Agroindústria de Alimentos) a $80{ }^{\circ} \mathrm{C}$ por 15 segundos; em seguida, a amostra foi armazenada em vidros e congelada para posterior avaliação da presença ou não de fungos termorresistentes.

\subsection{Análise de fungos termorresistentes}

Para enumeração dos fungos termorresistentes, utilizou-se o método de plaqueamento (PITT et al., 1979), já que ele recupera maior quantidade de esporos quando comparado ao método de incubação direta.

Foram transferidos assepticamente $100 \mathrm{~g}$ das polpas, provenientes das várias etapas de processamento, para saco estéril (Whirl-Pak®). $\mathrm{O}$ pH das amostras foram aferidos e ajustados quando necessário, para que fosse alcançado um valor final de 3,5. 
A amostra foi colocada em banho-maria a $80^{\circ} \mathrm{C}$ por 30 minutos e em seguida em banho de gelo por 15 minutos. Após o choque térmico, foram adicionados à amostra, $100 \mathrm{~mL}$ de meio Ágar Batata Dextrose (BDA) adicionado de cloranfenicol a $2 \mathrm{~g} / \mathrm{L}$ e ácido tartárico 10\% p/v.

O volume total da amostra, acrescido do meio BDA, foi vertido em 8 (oito) placas de $90 \times 15$ $\mathrm{mm}$ ou 4 placas de $120 \times 20 \mathrm{~mm}$. Após a solidificação do meio, as placas foram vedadas com Parafilm ${ }^{\circledR} \mathrm{M}$, armazenadas em sacos plásticos para evitar ressecamento, e incubadas por 30 dias a 30 ${ }^{\circ} \mathrm{C}$. Após esse período, foi realizada a leitura das placas.

\section{RESULTADOS E DISCUSSÃO}

\subsection{Análises microbiológicas}

Os resultados das análises microbiológicas realizadas nos frutos de cupuaçu estão de acordo com o padrão da legislação brasileira vigente, descritos na Tabela 2 e 3, para polpas de frutas no tocante a bolores e leveduras Coliformes $35^{\circ} \mathrm{C}$ e $45^{\circ} \mathrm{C}$ e Salmonella sp, tanto para safra de 2008 quanto para de 2010, o que era esperado, uma vez que a casca preserva a integridade do fruto, formando uma barreira e impedindo a entrada de microrganismos, por ser uma estrutura rígida e espessa.

Tabela 2. Resultados das análises microbiológicas realizadas na polpa de cupuaçu safra 2008

\begin{tabular}{ccccccc}
\hline \multicolumn{7}{c}{ Etapas de Processamento } \\
\hline $\begin{array}{c}\text { Análises } \\
\text { Microbiológicas }\end{array}$ & R & H & P & E & A & $\begin{array}{c}\text { Limites } \\
\text { Estabelecidos }\end{array}$ \\
\hline $\begin{array}{c}\text { Bolores e } \\
\text { Leveduras } \\
\text { (UFC/g)* }\end{array}$ & $<10$ & $4,8 \times 10$ & $<10$ & $4,4 \times 10$ & $1,6 \times 10$ & $\begin{array}{c}5 \times 10^{3} \text { (in } \\
\text { natura) }\end{array}$ \\
& & & & & & $2 \times 10^{3}$ \\
(processada)
\end{tabular}

*Estimado contagem inferior a 25 colônias e superior a 250 colônias. (-) ausência em 25 g. 
Tabela 3. Resultados das análises microbiológicas realizadas na polpa de cupuaçu safra 2010

\begin{tabular}{ccccccc}
\hline \multicolumn{7}{c}{ Etapas de Processamento } \\
\hline $\begin{array}{c}\text { Análises } \\
\text { Microbiológicas }\end{array}$ & R & H & P & E & A & $\begin{array}{c}\text { Limites } \\
\text { Estabelecidos }\end{array}$ \\
\hline $\begin{array}{c}\text { Bolores e } \\
\text { Leveduras } \\
\text { (UFC/g)* }\end{array}$ & $<10$ & $3,3 \times 10^{\mathrm{x}}$ & $8,3 \times 10^{\mathrm{x}}$ & $8,5 \times 10^{\mathrm{x}}$ & $9,8 \times 10^{\mathrm{x}}$ & $\begin{array}{c}5 \times 10^{3} \text { (in } \\
\text { natura })\end{array}$ \\
$\begin{array}{c}\text { Coliformes a } 35^{\circ} \mathrm{C} \\
(\mathrm{NMP} / \mathrm{g})\end{array}$ & $<3$ & $<3$ & $<3$ & $<3$ & $<3$ & $2 \times 10^{3}$ \\
$\begin{array}{c}\text { Coliformes a } 45^{\circ} \mathrm{C} \\
(\mathrm{NMP} / \mathrm{g})\end{array}$ & $<3$ & $<3$ & $<3$ & $<3$ & $<3$ & 1,0 \\
$\begin{array}{c}\text { Salmonella } \text { sp. } \\
\text { (processada) }\end{array}$ & - & - & - & - & - & - \\
\end{tabular}

*Estimado contagem inferior a 25 colônias e superior a 250 colônias. (-) ausência em 25 g.

Em qualquer das etapas de processamento nas quais foram coletadas amostras, não houve multiplicação de Coliformes a $35^{\circ} \mathrm{C}$ ou Coliformes a $45^{\circ} \mathrm{C}$, ou presença de Salmonella sp. Bueno et al. (2002) realizaram uma análise da qualidade microbiológica de 15 marcas de polpas de frutas congeladas incluindo cupuaçu, concluindo que todas as amostras atenderam à legislação vigente. Fungos filamentosos estavam presentes em todas as etapas do processamento, porém na ordem de $10^{1}$, enquanto o limite estabelecido pela legislação brasileira é na ordem de $10^{3}$ (Bueno et al. 2002).

A baixa contagem microbiana na polpa de cupuaçu pode ser atribuída à boa qualidade da matéria-prima empregada na fabricação do produto e da destruição de microrganismos promovida pela pasteurização, resfriamento e congelamento subsequentes (FAZIO et al., 1999). O cupuaçu tem ainda a característica físico-química de acidez elevada (faixa de $\mathrm{pH}$ situa-se entre 2,0 e 4,0) na polpa (FREIRE et al., 2009), o que restringe a flora microbiana capaz de se desenvolver com maior facilidade no meio.

Esses resultados mostraram boas condições higiênico-sanitárias no processamento da polpa de cupuaçu indicando a correta aplicação das boas práticas de fabricação (BPF). Em relação ao tempo de armazenamento, todas as etapas permaneceram estáveis microbiologicamente, apresentando ausência de Coliformes fecais $(<3 \mathrm{NMP} / \mathrm{mL}$ ) e de Salmonella durante os 3 meses de armazenamento, de acordo com os padrões microbiológicos estabelecidos para a polpa de fruta: coliformes fecais, máximo de 102/g e Salmonella, ausente em 25g. Em seus estudos, Alexandre et al. (2004), obtiveram resultados semelhantes, porém para o tempo de armazenamento de 5 meses.

Os valores obtidos neste trabalho para bolores e leveduras mostraram que as polpas homogeneizadas, pasteurizadas e envasadas estão de acordo com o padrão máximo estabelecido para bolores e leveduras. Os resultados obtidos por Santos et al. (2004) igualmente atenderam aos padrões legais estabelecidos pelo Ministério da Agricultura e do Abastecimento, tendo obtido contagens de bolores e leveduras inferiores a 10 UFC.g $\mathrm{g}^{-1}$ para polpa de cupuaçu congelada. Já Costa et al. (2003) encontraram contagens equivalentes a $1,1 \times 10^{2}$ UFC.g-1, em níveis de fungos filamentosos e leveduras próximos àqueles verificados por Freire et al. (2009). Por outro lado, Nascimento et al. (1999), ao analisarem o perfil microbiológico de polpas produzidas e comercializadas na cidade de São Luís MA, constataram que $100 \%$ das amostras apresentaram contaminação elevada por fungos filamentosos e leveduras, apresentando contagens entre $1,0 \times 10^{5} \mathrm{e} 1,1 \times 10^{8} \mathrm{UFC}_{\mathrm{g}} \mathrm{g}^{-1}$. 


\subsection{Análise de fungos termorresistentes}

As análises de fungos termorresitentes, realizadas para as polpas de cupuaçu etapa de processamento de homogeinização $(\mathrm{H})$ e etapa de processamento de pasteurização (P) (Figura 1), mostraram que os fungos sobreviveram ao processamento térmico, indicando que a pasteurização aplicada pela indústria não foi eficiente.

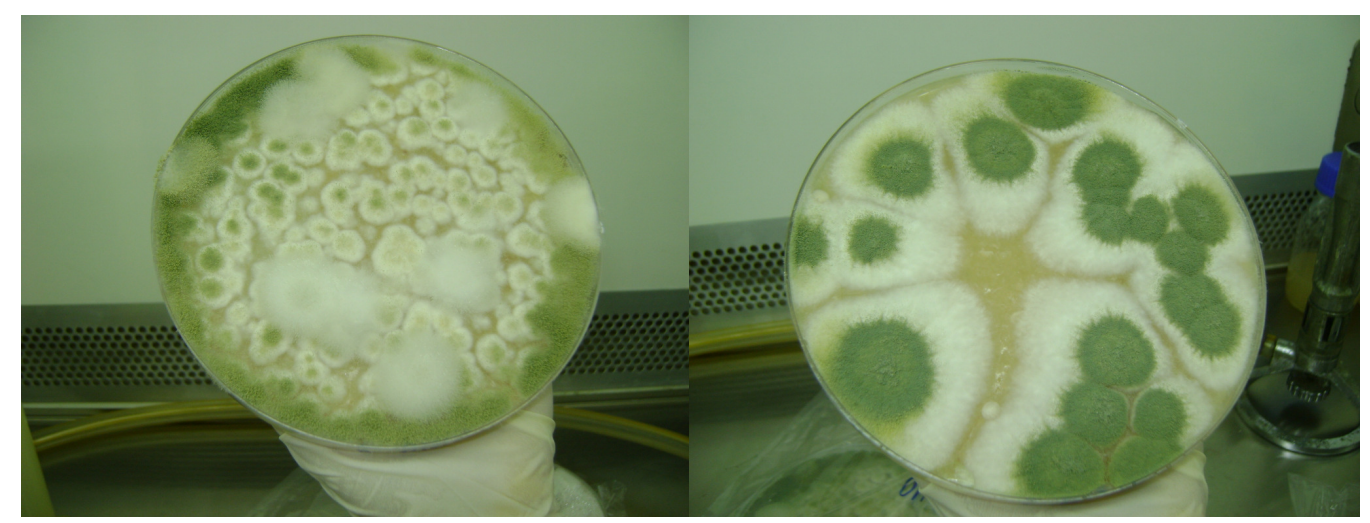

Figura 1. Crescimento de fungo termorresistente na polpa de cupuaçu proveniente das etapas de processamento: homogeinização $(\mathrm{H})$ e pasteurização $(\mathrm{P})$

A origem da contaminação da polpa de cupuaçu com fungos termorresistentes pode estar associada à contaminação inicial das frutas destinadas à produção, principalmente, com fungos originários do solo, facilmente introduzidos na manipulação para o processamento da polpa, uma vez que o cupuaçu é normalmente recolhido do chão e levado para o processamento na indústria. Quadro semelhante foi constatado por Pitt e Hocking (1985), em seus estudos sobre o maracujá, que também apresenta grande suscetibilidade de contaminação por ser diretamente recolhido e destinado ao processamento após cair no solo. Outros trabalhos estudam esse tipo de contaminação, tais como, Neosarthorya fischeri, isolado de morangos enlatados (McEVOY e STUART, 1970), de suco concentrado de abacaxi e de maracujá congelados (TOURNAS e TRAXLER, 1994).

Gumerato (1995) isolou o fungo Neosartotya fischeri presente em polpa de maçã, sendo que o mesmo microrganismo foi isolado na matéria prima, e no concentrado de maçã, mesmo depois deste último produto ter passado por processos térmicos da pasteurização e concentração, demonstrando potencial de resistência térmica do isolado. Algumas medidas podem ser aplicadas para minimizar a contaminação das frutas com fungos termorresistentes. Durante a colheita, deve-se evitar o contato das frutas com o solo, onde os ascósporos estão presentes. Antes do processamento, as etapas de lavagem com sanitizantes à base de cloro e a separação das frutas com deterioração visível contribuem significativamente na redução do número de ascósporos no produto final (HASAN, 2000).

$\mathrm{Na}$ análise feita com a polpa de cupuaçu proveniente da etapa de processamento $(\mathrm{H})$ devidamente pasteurizada em planta piloto II da Embrapa Agroindústria de Alimentos, não houve crescimento do fungo termorresistente (Figura 2), mostrando que o mesmo procedimento utilizado (80 ${ }^{\circ} \mathrm{C} / 15 \mathrm{~s}$ ) pela industria fornecedora da polpa de cupuaçu, talvez devido a ineficiência na limpeza e sanitização dos equipamentos e utensilhos, pode não ter sido eficiente. 


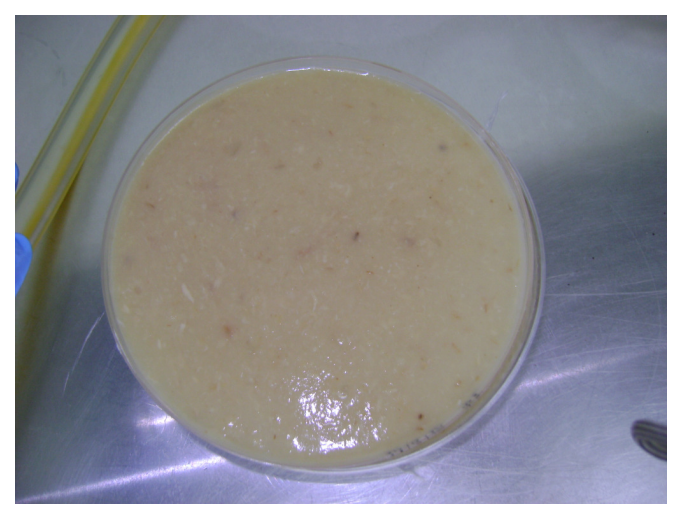

Figura 2. Crescimento de fungo termorresistente na polpa $\mathrm{R}$ de cupuaçu, pasteurizada em planta piloto II da Embrapa Agroindústria de Alimentos

Os bolores termorresistentes em frutas e derivados, apresentam elevada termorresistência nos produtos ácidos, isso se deve ao fato de alguns gêneros como Byssochlamys spp serem capazes de crescer sob baixas tensões de oxigênio deteriorando o produto (MAIA, 2007). Entretanto, é importante considerar que algumas vezes a extensão do crescimento destes fungos pode ser tão limitada que colônias ou material micelial podem não ser detectados visualmente, mesmo havendo deterioração evidente do produto. A comprovação nestes casos, pode ser feita por meio da detecção e enumeração de bolores termorresistentes, usando metodologia apropriada (TOURNAS, 1994).

\section{CONCLUSÃO}

A efetiva implantação das Boas Práticas de Fabricação aliada às características inerentes da polpa de cupuaçu e ao processo de pasteurização, foi suficiente para garantir a ausência de bactérias do grupo coliformes e Salmonella sp na polpa produzida na Embrapa Agroindústria de Alimentos durante as várias etapas de processamento e período de armazenamento.

A pasteurização empregada durante a produção da polpa de cupuaçu na indústria não foi eficiente para eliminar fungos termorresistentes. Esse fato implica em possível deterioração da polpa durante o armazenamento, considerando o quão é importante controlar a contaminação das frutas através da aplicação de boas práticas agrícolas, principalmente durante a colheita, transporte e armazenamento.

\section{REFERÊNCIAS BIBLIOGRÁFICAS}

ALEXANDRE, D., CUNHA, R.L., HUBINGER, M.D. Conservação do açaí pela tecnologia de obstáculos. Ciências e Tecnologia de Alimentos, Campinas, v. 24, n.1, p. 114-119, 2004.

APHA; Standard Methods for Examination of Water and Wastewater (APHA, AWWA), 20th ed., WPCF: New York, 2010.

BRASIL, Agência Nacional de Vigilância Sanitária - ANVISA. Resolução RDC - 12, 2 de Janeiro de 2001. Regulamento Técnico sobre Padrões Microbiológcios para Alimentos. Diário Oficial da União de 10/01/2001, Brasília - DF. Disponible in: <http:// www.anvisa.gov.br/e-legis/>. Acessed in: 10 Jun. 2009.

BUENO, S.M.; LOPES, M.R.V.; GRACIANO, R.A.S.; FERNANDES, E.C.B. \& GARCIA-CRUZ, C.H. Avaliação da polpa de frutas congeladas. Revista instituto Adolfo Lutz. v. 62, n.2, p. 121-126, 2002. 
COSTA, M. C.; MAIA, G. A.; SOUZA, M. S. M. F.; FIGUEIREDO, R. W.; NASSU, R. T.; MONTEIRO, J. C. S. Conservação de polpa de cupuaçu [Theobroma grandiflorum (Willd. Ex Spreng.) Schum] por métodos combinados. Revista Brasileira de Fruticultura, Jaboticabal, v. 25, n. 2, p. 213-215, 2003.

FAZIO, M.N.S.; GONÇALVES, T.M.V.; REPISSO, C.S.; MARTINS, M. \& HOFFMAN, F.L. Qualidade microbiológica de polpas congeladas de frutas, comercializadas na região de São José do Rio Preto, SP. Higiene Alimentar. v. 13, n. 66/67, p. 111-115, 1999.

FREIRE, M.T.A; PETRUS, R.R.; FREIRE, C.M.A; OLIVEIRA, C.A.F; FELIPE, A.M.P.F. \& GATTI, J.B. Caracterização físico-química, microbiológica e sensorial de polpa de cupuaçu congeladas (Theobroma grandiflorum Schum).Brazilian Journal of Food Technology. n 12, pp 9-16, 2009.

FOSTER, T. e VASAVADA, P. C. Beverage Quality and Safety. Institute of Food Technologists. [S.I.]: CRC Press. 248p. 2003.

GUMERATO, H. F. Desenvolvimento de um programa de computador para identificação de alguns fungos comuns em alimentos e determinação da resistência térmica de Neosartorya fischeri isolado de maçãs, 1995. 106 f. Tese (Doutorado em Tecnologia de Alimentos) - Faculdade de Engenharia de Alimentos, Universidade Estadual de Campinas, 1995.

HASAN, H. A. Patulin and aflatoxin in brown rot lesion of apple fruits and their regulation. World Journal of Microbiology and Biotechnology, v. 16, n. 7, p. 607-612, 2000.

MAIA, G. A.; SOUSA, P. H. M.; LIMA, A. S. L. Processamento de Frutas Tropicais. Fortaleza: Edições UFC, 320 p, 2007.

MAPA - Ministério da Agricultura, Pecuária e Abastecimento. Brasil. Instrução Normativa n.01 de 07 de janeiro de 2000. Aprovar o Regulamento Técnico Geral para fixação dos Padrões de Identidade e Qualidade para polpa de fruta. Diário Oficial da União.

MARTINS, V. B. Perfil sensorial de suco tropical de cupuaçu (Theobroma grandiflorum Schum) com valor calórico reduzido. 2008. 141 p. Tese (Doutorado em Alimentos e Nutrição) - Faculdade de Engenharia de Alimentos, Universidade Estadual de Campinas, Campinas, 2008.

McEVOY, I. J. \& STUART, M. R. Temperature tolerance of Aspergillus fischeri var glaber in canned strawberries. Irish Journal of Agricultural Research, Dublin, v. 9, p. 59-67, 1970.

NASCIMENTO, A. R. et al. Perfil microbiológico de polpas de acerola (Malpighia glabal) e abacaxi (Ananas comosus), produzidas e comercializadas na ilha de São Luís, MA. Higiene Alimentar, São Paulo, v. 13, n. 62, p. 44-47, 1999.

PITT, J.L. The genus Penicillium and its Teleomorphic States Eupenicillium and Talaromyces. 1st ed. London: Academic Press, 1979.

PITT, J.I. \& HOCKING, A.D. Fungi and food spoilage. Sydney: Academic Press, 1985, 413p.

SANTOS, F. A.; SALLES, J. R. J.; CHAGAS FILHO, E. \& RABELO, R. N. Análise qualitativa das polpas congeladas de frutas produzidas pela SUFRUTS, MA. Revista Higiene Alimentar, v. 18, n. 119, p.14-22, 2004.

TOURNAS V. Heat-resistant fungi of importance to the food and beverage industry. Critical Reviews in Microbiology, v. 20, n.4, p. 243-263, 1994. 
TOURNAS, V.; TRAXLER, R. W. Heat resistance of a Neosartorya fischeri strain isolated from pineapple juice frozen concentrate. J. Food Protect., v.57, n.9, p.814-816, 1994.

XAVIER, I. F. et al. Qualidade pos-colheita da manga Tommy Atkins $\square$ comercializada em diferentes estabelecimentos comerciais no municipio de Mossoro- RN. Revista Caatinga, Mossoro, v. 22, n. 4, p. 7- 13, 2009.

YANG, H.; PROTIVA, P.; CUI, B.; MA,C.; BGGETT, S.; HEQUET, V.; MORI, S.; WEINSTEIN, I.B. \& KENNELLY, E.J. New Bioactive Polyphenols from Theobrama grandiflorum ("Cupuaçu"). Journal of Natural Products. n 66, p.1501-1504, 2003. 